\title{
State-of-the-art in Reproductive Bench Science: Hurdles and New Technological Solutions
}

\author{
Felgueiras J. ${ }^{1}$, Ribeiro R. ${ }^{1}$, Brevini TAL. ${ }^{2}$, Costa P.F. ${ }^{1 *}$ \\ ${ }^{1}$ BIOFABICS - 3D Biotissue Analogues, R. Alfredo Allen 455, 4200-135 Porto, Portugal \\ 2 Laboratory of Biomedical Embryology, Centre for Stem Cell Research, Università degli \\ Studi di Milano, Milan, Italy \\ *pedro.costa@biofabics.com
}

Abstract:

global disability according to the World Health Organization. To study infertility and other reproductive system complications, bench science still relies on 2D and animal studies, which regularly have been criticized due to their inability to mimic the human body. Particular challenges in 2D studies include the inability to mimic fluid dynamics, gametes modulation and their crosstalk, hormonal patterns as well as the low quality and viability of gametes and embryos. Animal models also present other drawbacks, namely the absence of menstruation, making it difficult to establish a reliable predictive model for the human system. Additionally, reproductive studies should not be limited to the fallopian tube as the sole responsible for most infertility cases, but instead the research spectrum should be widened to the whole reproductive system given the tight interconnectivity between each and every organ. In the last few decades, new in vitro technologies have been developed and applied to the study of reproductive system complications. These systems allow to create complex three-dimensional structures, which are therefore able to more closely resemble specific microenvironments and 
provide more realistic physical and biochemical cues. 3D (bio)printing, organoids and

organs-on-chips are some of the dynamic technologies which are replacing conventionally employed static 2D culture. Herein, we provide an overview of the challenges found in conventional 2D and animal models of the reproductive system and present potential technological solutions for those same challenges.

Keywords: Infertility; Reproductive challenges; Biofabrication; Additive Manufacturing; Organs-on-chips.

\section{Introduction}

Infertility is defined as the inability to achieve clinical pregnancy after one year of regular unprotected intercourse and poses as a global public health issue [1]. According to the World Health Organization (WHO), about one in every four couples have been affected by infertility in developing countries [2]. This problem has a major impact not only on public human health, but also on the livestock industry, animal husbandry, and world food production. In this respect, abnormal ovulation and tubal obstruction are the main problems in female infertility, which lead to deficient fertilization as the oocyte is not able to correctly await fertilization in the fallopian tube [3]. Cancer is also correlated to infertility due to the gonadotoxic properties of some anticancer treatments [4].

The female reproductive system is mainly composed by ovaries, fallopian tubes, oviduct, uterus, and cervix [5]. Each organ is dynamic, responding to fluctuating hormonal concentrations driven by the pituitary gland and ovaries. This influences the ovulation, fertilization, embryo implantation, and placentation, making the reproductive system a 
highly complex system to study [6]. The main research and clinical reproductive advances were achieved so far by means of bi-dimensional (2D) in vitro culture studies, which will be later discussed. However, this type of model presents problems related to the lack of mimicry of the physiological system. Operative conditions such as $\mathrm{pH}$, osmolarity, light availability as well as availability of specific cues and factors are among the factors that make 2D in vitro studies inadequate [7]. Inappropriate culture conditions and resulting inadequate models can be detrimental and be a limitation in this research field. To our best knowledge, currently, most studies have a limited scope, focusing on studying mainly the oviduct and neglecting the bigger picture. However, it is indispensable to have a global perspective, to be able to understand the whole process and comprehend its dynamics.

Gamete/embryo handling and cell culture media can also be a limiting factor. For instance, the medium typically employed in 2D culture of embryos made by in vitro fertilization (IVF) procedures can effectively impact birth weight [8]. Despite all the advances in reproductive biotechnology, current operative conditions do not entirely mimic interactions and hormonal patterns observed in the natural morpho- physiological environment [9]. It is clear that in vitro-produced embryos differ markedly from those that have been developed in vivo, due to embryo manipulation and due to the static nature of cultures [10].

Nonetheless, when studying human fertility, animal models are not sufficiently useful either, since a reliable comparison cannot be made between these two systems. Animal models continue to face several challenges given that they are not entirely predictive of the human body function and there still are some safety and efficacy issues related to them [7]. Additionally, these models are significantly different from the human in vivo conditions, since most of them do not menstruate nor have the same regulatory patterns 
or specific hormones [11]. Rodents in particular are also insensitive to certain classes of chemical compounds, compromising the conclusiveness of any toxicological risk assessment intended to predict effects in humans [11].

In the present work we review various limitations encountered in reproductive research, as well as various novel technological solutions which may help advance the field.

\section{Conventional 2D cell culture and common hurdles in reproductive research}

In vitro static $2 \mathrm{D}$ cell culture is the most widely used strategy in the study of the female tract. It is a low cost, simple and convenient approach which also enables easy downstream processing $[12,13]$. A considerable number of related assays and techniques were also extensively developed turning $2 \mathrm{D}$ cell culture into a flexible and quick platform that can be easily employed in reproductive studies. In conventional 2D culture, cells are seeded on a plastic surface and allowed to grow in a bidirectional manner, having access to both media and growth factors $[12,13]$. The plastic surface can also be further functionalised with different materials and proteins in order to resemble certain microenvironments [14].

However, various studies alerted the scientific community to the sub-optimal conditions provided by 2D cell cultures. Particularly, 2D cell culture models can compromise the viability and reliability of experiments by influencing fundamental cellular features and in this way affect the correct understanding of the whole organ function $[12,13]$. During bidimensional growth, cells can suffer membrane receptor loss, modifications and alterations in several characteristics, such as genetic patterns, 
hormonal responses, cell differentiation and proliferation rates as well as responses to different stimuli and secretions [15]. Additionally, during manipulation of biological samples, these can suffer several manipulations, causing excessive stress to oocytes or embryos (temperature, light, $\mathrm{pH}$ and osmolarity) and compromising their potential $[8,16]$. Furthermore, in vitro 2D cultures showed to not be adequate for mimicking natural cellular environments given that the employed cellular monolayer suffers a quick transformation, making it difficult to maintain nutrient concentration and prevent metabolite accumulation $[16,17]$. This phenomenon is observable in cuboidal-columnar oviduct epithelial cells which change their phenotype into flattened cells with a complete loss of cilia and a reduced secretory ability, when compared to the normal physiology (figure 1) [16]. Inadequacies may also be found in 2D ovary cultures, which are not able to promote ovary follicle maturation due to the inexistence of follicular architecture. Another limitation found in 2D cell cultures is related to the inability to create a sufficient air-liquid interface, given that high volumes of medium are needed to supply proper conditions and most current embryo culture systems are static. Therefore, these systems often lose cell viability, compromising the experimental efficacy [10].

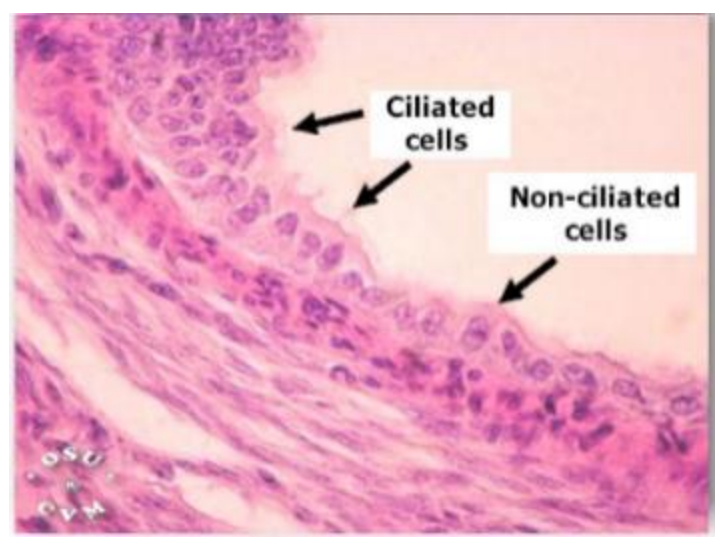

Figure 1 - Histological characterization of rat oviduct. Source: [78] 

cell culture is the lack of naturally occurring interactions between cells and organs. The oviduct is a good example of a non-reproducible system via 2D cell culture. This organ is characterised by highly complex interactions, being where the fertilization occurs and where the embryo develops before migrating to the uterus as a morula. It has a specific microenvironment that activates gametes, helps avoid polysemic fertilization, promotes embryo nourishment and provides developmental stimuli. Also, the oviduct wall is able to recognize the arrival of spermatozoa and even alter their proteome, due to these interactions $[7,18-21]$.

In vivo hormonal patterns are also impossible to be fully emulated in 2D cell cultures.

The long- and short-range hormonal signals that are established between the gametes or patterns. These signals promote a unique dialogue and generate multiple signalling cascades and a complex interactome, which may influence the maturation and transport of gametes, coordinating a successful fertilization and assuring embryo viability [20].

Genetic patterns are another hurdle found in 2D cell culture, given that many critical interactions that impact cells at a molecular level cannot be mimicked. The oviduct in particular has a specific role in embryonic genome activation and reprograming. These crucial interactions are responsible for creating somatic epigenetic methylation, which in turn modifies the gene expression by silencing or activating their expression through the epigenetic marks which later influence the blastocyte's transcriptome [22-24]. Ferraz et al. performed a comparative study between in vitro and in vivo embryos which showed that active DNA demethylation was higher in in vivo zygotes [15]. 

mechanical stresses are also factors that cannot be mimicked in $2 \mathrm{D}$ in vitro assays. When sensed, these forces are converted by cells into inner biochemical signals which influence their development. In particular, embryonic and extra-embryonic fluids have a critical role in the embryo behaviour since they modulate tensional and frictional stress and hydrostatic pressure [23]. Shear stress is one of the mechanical stresses that result from fluid flow, peristaltic tubal compression and kinetic friction between embryo and cilia, and which influence early development of the embryo [25-27]. This mechanical property is responsible for inducing cell-cell communication, positively affecting the embryo by refreshing the surrounding fluid, eliminating metabolites produced by the embryos and influencing genetic factors. Furthermore, mechanical events, such as pulsating muscle contractions, cilia beating and sperm motility, act in a positive way by increasing the dispersion and availability of hormones and nutrients [26]. Shear stress can modulate several important developmental mechanisms in pre-implanted embryos and should be recreated in in vitro experiments. along the tract and the interaction between the different female organs. Such limitations may affect not only the gametes but also the embryo in their DNA methylation dynamics or hormonal signalling. As such, all these experimental conditions may impact the embryo's outcome and consequently promote sub-optimal conditions in the reproductive system studies. 

complex approaches to investigate the female reproductive system. These allow to more closely mimic the human body, having the potential to address the limitations previously mentioned. Three-dimensional (3D) cell culture, organoid models and organ-on-chip are some of these new advanced systems, which will be discussed below.

3D cell culture systems emerged as a way to overcome the hurdles reported above, given their ability to more closely mimic the macro- and micro-architecture of tissues and organs, and to stimulate cells with the appropriate biochemical and biomechanical cues. The transition from 2D to 3D models represents a breakthrough in cell biology and related areas since it can lead to levels of tissue organisation never seen before. In these cell culture systems, cells grow and organize themselves in a 3D architecture, promoting a more complex structures and representing a more in vivo-like biological 172 microenvironment. In this way, the three-dimensionality improves and promotes communication between cells, replicating cell and tissue physiology, mimicking mechanical cues, allowing communication between the cell and its matrix and taking into account the spatial organization of the tissue [12-14]. Moreover, 3D approach allows cells to maintain the basal-apical polarity, as well as to retain their genetic and epigenetic patterns. However, these models may in certain cases still face some challenges involving deficient non-homogenous distribution of cells, nutrients and oxygen, inefficient removal of waste, lack of vascularisation and therefore limited reliability and repeatability [28]. will be described below. 
Spheroids are simple 3D models that take advantage of the capability of adherent cells to aggregate. These cell aggregates are able to mimic the microenvironment of various tissues as well as their cell-cell and cell-ECM interactions. However, the generation of necrotic cores is generally a considerable challenge of this technique, due to the lack of nutrients, oxygen and waste diffusion to/from the center of the cell aggregate. Due to its simplicity and mimicry properties, spheroids are widely used in drug screening assays [28]. Lawrenson et al have successfully developed a fallopian tube spheroid model composed of primary fallopian tube secretory epithelial cells. The employed spheroids were able to restore the three-dimensional in vivo architecture although at a low proliferative rate. In this way, it was possible to show that spheroid technologies are able to generate relevant models to study the quiescent status of normal secretory epithelial cells [29].

Microcapsules are mainly used as 3D cell carriers, and have been employed in the delivery of cells for the treatment of conditions such as cancer and diabetes [30-33]. Microcapsules are small sized systems, ranging from $100 \mu \mathrm{m}$ to $750 \mu \mathrm{m}$, generally made of natural or synthetic polymers and able to encapsulate hundreds to thousands of cells. The reduced capsule size allows to increase oxygen, nutrient and waste diffusion and allow delivery of cells and therapeutics via small incisions or catheters, therefore avoiding major surgeries $[32,33]$. Dorati et al developed a 3D barium-alginate microcapsule for enriching the medium employed in in vitro embryo production from cryopreserved domestic cat vitrified oocytes. The results of this study showed that while using vitrified oocytes 3D culture per se did not capacitate the viability of vitrified cat oocytes. However, 
when using a culture condition enriched with 3D alginate microcapsules, it was possible

to promote maturation and embryo development [34]. pluripotent stem cells or multipotent organ-specific adult stem cells [35]. These 3D models have a higher degree of complexity, and, therefore, are able to more reliably mimic tissue's histology, functionality and physiology, when comparing to the classical 2D in vitro cell culture. Additionally, organoids are able to retain tissue phenotypical and functional properties [36]. In a study by Kessler and co-workers it was possible to successfully produce a fallopian tube organoid made from stem cells derived from the human fallopian tube epithelium [37]. This model could mimic the normal physiology and anatomy of the human fallopian tube epithelium, maintain the phenotypic patterns for several months and differentiate epithelial cells into secretory and ciliated cells. Finally, the authors were able to observe a high degree of similarity between the organoid and its human counterpart. Also using an organoid model, Buretto et al created an endometrium model that could efficiently reproduce the tissue physiology and allow long-term expansion [36]. Despite all, organoid morphology and architecture are still a performed manually, therefore increasing the susceptibility to error [16].

Scaffolds are support constructs that foster cell and tissue growth. They can also further influence cell and tissue development by providing topographical cues and/or 
various biochemical cues, such as growth factors and drugs [38]. Scaffolds can be created

231

using different physical and/or chemical approaches and composed of a wide range of materials, either natural, synthetic or hybrid. Some of the most promising scaffolds are composed of hydrogels and manufactured by means of biofabrication techniques [39-41].

Hydrogels are three-dimensional networks composed of physically or chemically crosslinked hydrophilic polymers. These are biocompatible and, although being water insoluble, can uptake high amounts of water or other liquids and may have their physical conformation tuned in terms of mechanical and morphological properties $[42,43]$. The utilization of hydrogels may however be limited given that some of them are created employing harmful crosslinkers and most lack adequate mechanical and degradability properties. These limitations are usually overcome by combining both natural and synthetic polymers in order to obtain hydrogels with tailored properties [42-44]. Joo et al used a collagen-based hydrogel for seeding oocytes and investigate the hormone patterns and oocyte maturation. The employed hydrogel was shown to indeed contribute to the maintenance of follicle native function and its phenotypic patterns when cultured in vitro [45]. such as electrospinning, among others [46] . Due to the precision of these technologies, scaffolds can be manufactured with tailored morphology and mechanical properties, maximizing the biomimetic properties of these systems $[39,47]$. Furthermore, by employing these technologies it is possible to achieve greater levels of repeatability and reproducibility, when compared to other cell culture approaches [48]. However, similarly 
254 to other scaffolds, limited nutrient, oxygen and waste diffusion in large scaffold volumes,

255 heterogeneous cell distribution and architectural challenges are among the drawbacks

256 that may eventually be observed in these systems $[48,49]$. In a study by Liverani et al

257 employing electrospun fibrous scaffolds for culture of porcine follicles, it was possible to

258 observe that scaffold morphology and composition had crucial roles in enabling the ovary

259 to maintain its normal function and follicle morphology, given that the scaffold could

260 closely mimic the in vivo tissue [50].

\begin{tabular}{|c|c|c|c|}
\hline 3D MODELS & ADVANTAGES & DISADVANTAGES & REFERENCES \\
\hline SPHEROIDS & $\begin{array}{l}\text {-Simple } \\
\text {-Mimics in vivo interactions }\end{array}$ & $\begin{array}{l}\text {-Necrotic cores } \\
\text {-Size variability } \\
\text { - Long-term culture } \\
\text { difficulty } \\
\text {-Simplified architecture }\end{array}$ & {$[29,51-54]$} \\
\hline ORGANOIDS & $\begin{array}{l}\text {-Emulate interaction } \\
\text { between cells of different } \\
\text { tissues } \\
\text {-Higher degree of } \\
\text { complexity } \\
\text {-Can be patient specific }\end{array}$ & $\begin{array}{l}\text {-Necrotic cores } \\
\text {-Size variability } \\
\text {-Need validation }\end{array}$ & [54-56] \\
\hline 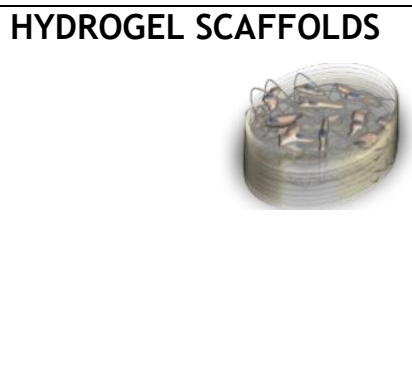 & $\begin{array}{l}\text {-Emulate in vivo ECM } \\
\text { interaction } \\
\text { - Physical or chemical } \\
\text { crosslinking } \\
\text { - Highy reproducible }\end{array}$ & $\begin{array}{l}\text {-Inefficient exchange of } \\
\text { substances } \\
\text {-Simplified architecture } \\
\text {-Difficult cell recovery }\end{array}$ & {$[43,54]$} \\
\hline
\end{tabular}




\begin{tabular}{|l|l|l|l|}
\hline BIOFABRICATED & - Mimics the in vivo & - Heterogeneous cell & {$[54,57,58]$} \\
SCAFFOLDS & distribution & \\
& - Hicroenvironment & - Limited diffusion \\
& -Tailored morphology and & & \\
& mechanical properties & & \\
\hline
\end{tabular}

262

263

Advanced technological solutions

264

Nowadays there are new advanced technologies that are capable of further

replicating the complex heterogeneity found in the reproductive system in a more precise

manner and therefore being able to closely resemble in vivo microenvironments. These

technologies include 3D printing and bioprinting, as well as microfluidic systems and organ-on-chips, which will be discussed below.

3D printed scaffolds and devices. 3D printing technology is based on the

fabrication of 3D structures by consecutive deposition of layers of material. It was first used in 1986 by Charles W. Hu, who first developed stereolithography [59]. By using computer-aided design (CAD) software and medical imaging, simple or complex physical shapes can be obtained with an unprecedented speed and detail. The development and optimisation of new materials, combined with reduced manufacturing costs and advanced printers, enabled the use of 3D printing in research labs and industrial settings

277 [60-62]. This technology can be adopted for a broad range of applications, from aviation and car industries to healthcare. In the medical field, the use of biocompatible materials, such as polylactic acid (PLA) and acrylonitrile butadiene styrene (ABS) allowed to produce scaffolds that could be used in certain medical scenarios [60-62]. Additionally, 3D printing presents a tremendous potential for scaffold production due to the increased reliability 
and reproducibility, when compared to other methods. Post-manufacturing treatments, such as heat treatments and hot isostatic pressing, are used to tailor the properties of scaffolds, by modifying their microstructure or surface roughness [62].

3D Bioprinting (a very specific type of 3D printing) also applies a layer-by-layer concept however, unlike standard 3D printing, it relies instead on the deposition of cellladen filaments or cell-containing droplets into specific substrates in order to generate three-dimensional biological structures [59].

Regarding the application of 3D printing in the study of the reproductive system, the best example can be found in a study by Laronda et al. describing the development of a bioprosthetic ovary. In this case, a 3D printed microporous scaffold was able to provide space and nutrition diffusion for follicle survival and maturation and enabled the growth of an ovary-like vascularization, which is fundamental for the circulation of hormones around the follicle. This system also enabled the development of a corpus luteus to produce hormones, after the ovulation, and egg release without mechanical manipulation or digestion [63].

(1)

Microfluidic systems are another strategy available to study the reproductive field in a more complex manner. Microfluidic systems are small devices with a single or a set of sub-millimetric channels. These micro-channels allow the injection of liquids or gases through inlets connected to tubing and/or syringe/pump adapters. Such devices can be used for several purposes not only in the biomedical field but also in chemistry and other areas. The utilization of microfluidics for reproductive purposes has increased in the past years due to their ability to mimic the reproductive system in a more efficient and physiological way [19]. Microfluidics has already been used on sperm motility tests 
allowing to study sperm rheotaxis and chemotaxis [64,65], gamete and embryo culture and evaluation $[23,66]$, transportation of embryos and respective monitoring $[66,67]$ and to remove cumulus cells and zona pellucida $[17,68]$. For example, Angione et al were able to develop a microfluidic device that not only allowed perfusion and live imaging of the living system but also a precise and flexible handling of individual oocytes and embryos [69]. Another example is the device developed by Yin et al. which could mimic an in vivolike 3D placenta in a chip [70]. In this study, the construct was designed with two parallel cell channels separated by a middle matrix channel, and with a perfusion flow that could generate a near-physiological dynamic microenvironment. In this way, it was possible to environments. on-chips may consist of very simple devices or highly complex device systems, often

Organs-on-chips are complex microfluidic devices, designed for emulating the architecture, function and dynamic environment of organs (or portions thereof). Organsintegrating components such as valves or pumps into the design itself. These systems can for instance allow fluid mixing, as well as generate fluid gradients and microdroplets [71]. Furthermore, the integration of flow perfusion and the ability to co-culture cells in a controlled manner makes these devices more able to resemble in vivo conditions.

Organs-on-chips are highly suitable for laboratory assays since they are small, employ low volumes of reagents being therefore less expensive to operate, and are able to generate and maintain a stable microenvironment with precise control over spatial and temporal dynamics. Additionally, these systems enable a greater capability for batch sample processing and greater screening scope $[19,72]$. These systems are already being 
used for medical and pharmaceutical purposes, namely in drug screening and

331 pharmacokinetic and pharmacodynamic tests [5]. These devices are also able to better

332 simulate natural environments which are useful for embryo culture optimization. Organson-chips can also mimic naturally occurring physical and mechanical stimuli, enabling cells

to experience relevant physiological cues that directly affect their biological function [73].

Additionally, medium perfusion allows to improve nutrient supply, clearance of waste products and metabolic profiling [73]. Furthermore, organs-on-chips are adequate for profiling the cell secretome in culture medium and, therefore, used for searching specific biomarkers [74]. In the future, this technology may be used as a fertilization platform, improving the in vitro fertilization rate and quality, as well as simultaneously integrate a variety of functional tests that supplement the information about embryos or gametes. Finally, by providing an environment with controlled osmolality, temperature, and $\mathrm{pH}$, organ-on-chip devices could eventually reduce the stress typically imposed on embryos and enable spermatozoa and oocyte interaction to take place in in vivo-like environments, generating embryos more suitable for implantation [19]. The most advanced examples of organ-on-chip devices directed at reproductive studies were developed by Ferraz et al. [21] and Xiao et al. [6].

In the first case, a polydimethylsiloxane (PDMS) microfluidic oviduct-on-chip device was developed, comprising two independent and perfusable $370 \mu \mathrm{m}$ deep compartments separated by a porous membrane. To mimic the oviduct, a confluent oviduct epithelial cell layer was grown on the top of the membrane. The compartments were designed to ensure shear stress and to entrap the oocytes in an apical compartment. This device was built with a thin design so that the apical compartment of the device would allow live imaging of the epithelial cells, gametes, and embryos inside the chip [21]. This 3D model 
allowed a deeper understanding in the early maternal-gamete/embryo interaction with production of zygotes highly resembling in vivo zygotes, within a microenvironment closely resembling in vivo conditions.

In the latter case, a complex multi-organ on chip (called EVATAR) was composed of five different tissues (cervix, fallopian tube, ovaries, uterus, and liver) connected by a complex microfluidic device circulating flow between all tissues. Given that the use of microfluidic devices enable the control of flow rates and real-time monitoring of metabolites, drug compounds, signalling molecules and hormones $[5,65]$, this device could more realistically mimic the complexity of the reproductive female system, when comparing to 2D petri dishes or animal models. Additionally, this organ-on-chip was able to mimic the 28-day in vivo human follicular and luteal phase hormone synthesis and hence enable the provision of steroid and peptide hormones [6]. Such systems represent the next step in the study of the female reproductive system and may become an $[75,76]$.

In general, such complex microfluidic platforms can help reveal more biological insights more reliably than usual lab assays, since they can recreate the physiological microenvironment, replicating many features such as chemical gradients, fluid dynamics, surface interactions and morphologies [75]. However, before widespread adoption, it is important to make sure that these devices are fully tested and characterized in order to safeguard the reliability of results obtained. As an example, the material most commonly 
used in the manufacture of microfluidic and organ-on-chip devices - PDMS - undesirably binds to some classes of molecules and some additive manufacturing materials may be toxic to cells [76].

\section{Conclusions and future perspectives}

Conventional 2D static culture methods are clearly uncapable of fully mimicking the natural environment where mammalian embryos typically develop under constant exposure to complex combinations of stimuli, crosstalk and cascade reactions. Advanced technologies such as organoids, bioprinting and organs-on-chips show the potential to address these limitations by ultimately enabling the creation of 3D devices and constructs where embryos and micro replicas of various reproductive organs and tissues may be grown in close communication with each other. The ability to spatially control the threedimensional positioning of all these elements as well as the ability to accurately control their ability to communicate amongst themselves provides a powerful means to perform systematic in-depth studies where the role of each of these elements can be elucidated and quantified as the embryo development progresses. Apart from simply mimicking normal physiological conditions, it may also become possible to induce abnormal stimuli and conditions, therefore enabling the study and understanding of diseased or abnormal states which, apart from rarely occurring in nature, may also be extremely difficult or even impossible to properly study in conventional in vivo and in vitro conditions.

Despite the unquestionable potential of these new technologies, it is however important to keep in mind that they are fairly recent and are still rarely employed in reproductive studies. In that sense, there is still a long and arduous way to go until such 
401 technologies become routinely employed for reproductive purposes. Not only the 402 technology needs to be further developed in order to achieve its full potential, but it also 403 needs to become standardized so that results obtained from different studies can be 404 adequately combined and compared. Furthermore, and given the dynamic complexity of 405 the biological systems generated in this way, it will be equally important to assure the quality and speed of acquisition of all sensorial and analytical methods employed. Overall, the new technologies herein described show the potential to revolutionize reproductive research as well as human and animal reproduction as a whole, although much work still needs to be done so that these technologies may become standardized and routinely adopted in the field.

\section{Acknowledgements}

413 This project has received funding from the European Union's Horizon 2020 research and 414 innovation programme under the Marie Sklodowska-Curie grant agreement No 798014.

415 This project has received funding from the European Union's Horizon 2020 research and innovation programme under grant agreement No 828835. and fitness (CellFit).

\section{Conflict of Interest}

Dr. Costa is CEO, CTO, and shareholder of the company Biofabics Lda. 


\section{References}

426

427

428

429

430

431

432

433

434

435

436

437

438

439

440

441

442

[1] "Infertility is a global public health issue." Available https://www.who.int/reproductivehealth/topics/infertility/perspective/en/ [2] "Global prevalence of infertility, infecundity and childlessness." Available https://www.who.int/reproductivehealth/topics/infertility/burden/en/

[3] W. L. Kao, H. Y. Huang, and D. J. Yao, “Oviduct-mimetic chip to improve in vitro fertilization for oligozoospermia," 9th IEEE Int. Conf. Nano/Micro Eng. Mol. Syst. IEEE-NEMS 2014, pp. 152-156, 2014.

[4] T. Iwamoto, "Clinical Application of Drug Delivery Systems in Cancer Chemotherapy : Review of the Efficacy and Side Effects of Approved Drugs," Biol. Pharm. Bulliten, vol. 36, no. May, pp. 715-718, 2013.

[5] J. Nawroth, J. Rogal, M. Weiss, S. Y. Brucker, and P. Loskill, “Organ-on-a-Chip Systems for Women's Health Applications," Adv. Healthc. Mater., vol. 7, no. 2, pp. 1-21, 2018.

[6] S. Xiao, J. R. Coppeta, H. B. Rogers, B. C. Isenberg, J. Zhu, S. A. Olalekan, K. E. McKinnon, D. Dokic, A. S. Rashedi, D. J. Haisenleder, S. S. Malpani, C. A. ArnoldMurray, K. Chen, M. Jiang, L. Bai, C. T. Nguyen, J. Zhang, M. M. Laronda, T. J. Hope, K. P. Maniar, M. E. Pavone, M. J. Avram, E. C. Sefton, S. Getsios, J. E. Burdette, J. J. Kim, J. T. Borenstein, and T. K. Woodruff, "A microfluidic culture model of the human reproductive tract and 28-day menstrual cycle," Nat. 
[7] K. Ronaldson-Bouchard and G. Vunjak-Novakovic, "Organs-on-a-Chip: A Fast Track for Engineered Human Tissues in Drug Development," Cell Stem Cell, vol. 22, no. 3, pp. 310-324, 2018.

[8] S. Li and W. Winuthayanon, "Oviduct: Roles in fertilization and early embryo development," J. Endocrinol., vol. 232, no. 1, pp. R1-R26, 2017.

[9] R. Ivell, "Research in reproduction: Challenges, needs, and opportunities," Front.

[10] M. A. M. M. Ferraz, H. H. W. Henning, P. F. Costa, J. Malda, F. P. Melchels, R. Wubbolts, T. A. E. Stout, P. L. A. M. Vos, and B. M. Gadella, "Improved bovine embryo production in an oviduct-on-a-chip system: prevention of poly-spermic fertilization and parthenogenic activation," Lab Chip, vol. 17, no. 5, pp. 905-916, 2017.

[11] A. D. van den Brand, E. Rubinstein, P. C. de Jong, M. van den Berg, and M. B. M. van Duursen, "Primary endometrial 3D co-cultures: A comparison between human and rat endometrium," J. Steroid Biochem. Mol. Biol., vol. 194, no. August, p. 105458, 2019.

[12] M. Kapałczyńska, T. Kolenda, W. Przybyła, M. Zajączkowska, A. Teresiak, V. Filas, M. Ibbs, R. Bliźniak, Ł. Łuczewski, and K. Lamperska, "State of the art paper 2D and 3D cell cultures - a comparison of different types of cancer cell cultures," 2018.

[13] K. Duval, H. Grover, L. H. Han, Y. Mou, A. F. Pegoraro, J. Fredberg, and Z. Chen, 
"Modeling physiological events in 2D vs. 3D cell culture," Physiology, vol. 32, no. 4, pp. 266-277, 2017.

[14] S. A. Langhans, "Three-dimensional in vitro cell culture models in drug discovery and drug repositioning," Front. Pharmacol., vol. 9, no. JAN, pp. 1-14, 2018.

[15] M. de Almeida Monteiro Melo Ferraz, Oviduct-on-a-chip : Creating an in vitro oviduct to study bovine gamete interaction and early embryo development. 2018.

[16] M. A. M. M. Ferraz, H. H. W. Henning, T. A. E. Stout, P. L. A. M. Vos, and B. M. Gadella, “Designing 3-Dimensional In Vitro Oviduct Culture Systems to Study Mammalian Fertilization and Embryo Production," Ann. Biomed. Eng., vol. 45, no. 7, pp. 1731-1744, 2017.

[17] H. C. Zeringue, J. J. Rutledge, and D. J. Beebe, "Early mammalian embryo development depends on cumulus removal technique," Lab Chip, vol. 5, no. 1, pp. 86-90, 2005.

[18] B. Fernandez-Fuertes, B. Rodríguez-Alonso, J. M. Sánchez, C. A. Simintiras, P. Lonergan, and D. Rizos, "Looking at the big picture: Understanding how the oviduct's dialogue with gametes and the embryo shapes reproductive success," Anim. Reprod., vol. 15, no. Irrs, pp. 751-764, 2018.

[19] Z. Peter Nagy, A. Varghese, and A. Agarwal, "In Vitro Fertilization A Textbook of Current and Emerging Methods and Devices: A Textbook of Current and Emerging Methods and Devices," Vitr. Fertil., pp. 625-642, 2019.

[20] A. Fazeli and E. Pewsey, "Maternal communication with gametes and embryos: A complex interactome," Briefings Funct. Genomics Proteomics, vol. 7, no. 2, pp. 
490

491

492

493

494

495

496

497

498

499

500

501

502

503

504

505

506

507

508

509

510

[21] M. A. M. M. Ferraz, H. S. Rho, D. Hemerich, H. H. W. Henning, H. T. A. van Tol, M. Hölker, U. Besenfelder, M. Mokry, P. L. A. M. Vos, T. A. E. Stout, S. Le Gac, and B. M. Gadella, “An oviduct-on-a-chip provides an enhanced in vitro environment for zygote genome reprogramming," Nat. Commun., vol. 9, no. 1, 2018.

[22] N. El Hajj and T. Haaf, "Epigenetic disturbances in in vitro cultured gametes and embryos: Implications for human assisted reproduction," Fertil. Steril., vol. 99, no. 3, pp. 632-641, 2013.

[23] N. Kashaninejad, M. J. A. Shiddiky, and N. T. Nguyen, "Advances in MicrofluidicsBased Assisted Reproductive Technology: From Sperm Sorter to Reproductive System-on-a-Chip," Adv. Biosyst., vol. 2, no. 3, pp. 1-21, 2018.

[24] K. Matsuura, N. Hayashi, Y. Kuroda, C. Takiue, R. Hirata, M. Takenami, Y. Aoi, N. Yoshioka, T. Habara, T. Mukaida, and K. Naruse, "Improved development of mouse and human embryos using a tilting embryo culture system," Reprod. Biomed. Online, vol. 20, no. 3, pp. 358-364, 2010.

[25] Y. Xie, F. Wang, W. Zhong, E. Puscheck, H. Shen, and D. A. Rappolee, "Shear Stress Induces Preimplantation Embryo Death That Is Delayed by the Zona Pellucida and Associated with Stress-Activated Protein Kinase-Mediated Apoptosis1," Biol. Reprod., vol. 75, no. 1, pp. 45-55, 2006.

[26] J. Solowiej-Wedderburn, D. J. Smith, S. S. Lopes, and T. D. Montenegro-Johnson, "Wall stress enhanced exocytosis of extracellular vesicles as a possible mechanism of left-right symmetry-breaking in vertebrate development," J. Theor. 

Biol., vol. 460, pp. 220-226, 2019.

[27] M. Torabi, M. Drahansky, M. . Paridah, A. Moradbak, A. . Mohamed, F. abdulwahab taiwo Owolabi, M. Asniza, and S. H. . Abdul Khalid, "We are IntechOpen, the world's leading publisher of Open Access books Built by scientists , for scientists TOP $1 \%$," Intech, vol. i, no. tourism, p. 13, 2016.

[28] R. Edmondson, J. J. Broglie, A. F. Adcock, and L. Yang, "Three-dimensional cell culture systems and their applications in drug discovery and cell-based biosensors," Assay Drug Dev. Technol., vol. 12, no. 4, pp. 207-218, 2014.

[29] K. Lawrenson, M. Notaridou, N. Lee, E. Benjamin, I. J. Jacobs, C. Jones, and S. A. Gayther, "In vitro three-dimensional modeling of fallopian tube secretory epithelial cells," BMC Cell Biol., vol. 14, no. 1, p. 1, 2013.

[30] S. Chang and T. European, "<Cell-encapsulation-Promise-andprogress_2003_Nature-Medicine.pdf>," vol. 9, no. 1, 2003.

[31] G. Orive, E. Santos, D. Poncelet, R. M. Hernández, J. L. Pedraz, L. U. Wahlberg, P. De Vos, and D. Emerich, "Cell encapsulation: technical and clinical advances," Trends Pharmacol. Sci., vol. 36, no. 8, pp. 537-546, 2015.

[32] A. Murua, A. Portero, G. Orive, R. M. Hernández, M. de Castro, and J. L. Pedraz, "Cell microencapsulation technology: Towards clinical application," J. Control. Release, vol. 132, no. 2, pp. 76-83, 2008.

[33] J. M. Rabanel, X. Banquy, H. Zouaoui, M. Mokhtar, and P. Hildgen, "Progress technology in microencapsulation methods for Cell therapy," Biotechnol. Prog., vol. 25, no. 4, pp. 946-963, 2009. 
[34] R. Dorati, I. Genta, M. Ferrari, G. Vigone, V. Merico, S. Garagna, M. Zuccotti, and B. Conti, "Formulation and stability evaluation of 3D alginate beads potentially useful for cumulus-oocyte complexes culture," J. Microencapsul., vol. 33, no. 2, pp. 137-145, 2016.

[35] K. Kretzschmar and H. Clevers, "Organoids: Modeling Development and the Stem Cell Niche in a Dish," Dev. Cell, vol. 38, no. 6, pp. 590-600, 2016.

[36] M. Boretto, B. Cox, M. Noben, N. Hendriks, A. Fassbender, H. Roose, F. Amant, D. Timmerman, C. Tomassetti, A. Vanhie, C. Meuleman, M. Ferrante, and H. Vankelecom, "Development of organoids from mouse and human endometrium showing endometrial epithelium physiology and long-term expandability," Dev., vol. 144, no. 10, pp. 1775-1786, 2017.

[37] M. Kessler, K. Hoffmann, V. Brinkmann, O. Thieck, S. Jackisch, B. Toelle, H. Berger, H. J. Mollenkopf, M. Mangler, J. Sehouli, C. Fotopoulou, and T. F. Meyer, "The Notch and Wnt pathways regulate stemness and differentiation in human fallopian tube organoids," Nat. Commun., vol. 6, no. May, 2015.

[38] D. Howard, L. D. Buttery, K. M. Shakesheff, and S. J. Roberts, “Tissue engineering: Strategies, stem cells and scaffolds," J. Anat., vol. 213, no. 1, pp. 66-72, 2008.

[39] E. Sachlos, J. T. Czernuszka, S. Gogolewski, and M. Dalby, “Making tissue engineering scaffolds work. Review on the application ofsolid freeform fabrication technology to the production of tissue engineeringscaffolds," Eur. Cells Mater., vol. 5, pp. 29-40, 2003.

[40] P. Morouço, W. Lattanzi, and N. Alves, "Four-dimensional bioprinting as a new era 
for tissue engineering and regenerative medicine," Front. Bioeng. Biotechnol., vol. 5, no. OCT, pp. 1-3, 2017.

[41] I. M. El-Sherbiny and M. H. Yacoub, "Hydrogel scaffolds for tissue engineering: Progress and challenges," Glob. Cardiol. Sci. Pract., vol. 2013, no. 3, p. 38, 2013.

[42] R. A. Batista, C. G. Otoni, and P. J. P. Espitia, Fundamentals of chitosan-based hydrogels: elaboration and characterization techniques, vol. 1. Elsevier Inc., 2019.

[43] E. M. Ahmed, “Hydrogel: Preparation, characterization, and applications: A review," J. Adv. Res., vol. 6, no. 2, pp. 105-121, 2015.

[44] U. Freudenberg, Y. Liang, K. L. Kiick, and C. Werner, "Glycosaminoglycan-based biohybrid hydrogels: a sweet and smart choice for multifunctional biomaterials," Adv. Mater., no. 28, pp. 8861-8891, 2016.

[45] S. Joo, S. H. Oh, S. Sittadjody, E. C. Opara, J. D. Jackson, S. J. Lee, J. J. Yoo, and A. Atala, "The effect of collagen hydrogel on 3D culture of ovarian follicles," Biomed. Mater., vol. 11, no. 6, 2016.

[46] I. Jun, H. S. Han, J. R. Edwards, and H. Jeon, "Electrospun fibrous scaffolds for tissue engineering: Viewpoints on architecture and fabrication," Int. J. Mol. Sci., vol. 19, no. 3, 2018.

[47] J. An, J. E. M. Teoh, R. Suntornnond, and C. K. Chua, "Design and 3D Printing of Scaffolds and Tissues," Engineering, vol. 1, no. 2, pp. 261-268, 2015.

[48] D. W. Hutmacher, Scaffolds in tissue engineering bone and cartilage, vol. 21. Woodhead Publishing Limited, 2000. 
[49] S. M. Giannitelli, D. Accoto, M. Trombetta, and A. Rainer, "Current trends in the design of scaffolds for computer-aided tissue engineering," Acta Biomater., vol. 10, no. 2, pp. 580-594, 2014.

[50] L. Liverani, N. Raffel, A. Fattahi, A. Preis, I. Hoffmann, A. R. Boccaccini, M. W. Beckmann, and R. Dittrich, "Electrospun patterned porous scaffolds for the support of ovarian follicles growth: a feasibility study," Sci. Rep., vol. 9, no. 1, pp. 1-14, 2019.

[51] T. H. Kim, J. H. Choi, Y. Jun, S. M. Lim, S. Park, J. Y. Paek, S. H. Lee, J. Y. Hwang, and G. J. Kim, “3D-cultured human placenta-derived mesenchymal stem cell spheroids enhance ovary function by inducing folliculogenesis," Sci. Rep., vol. 8, no. 1, pp. 1-11, 2018.

[52] A. Domnina, P. Novikova, J. Obidina, I. Fridlyanskaya, L. Alekseenko, I. Kozhukharova, O. Lyublinskaya, V. Zenin, and N. Nikolsky, "Human mesenchymal stem cells in spheroids improve fertility in model animals with damaged endometrium," Stem Cell Res. Ther., vol. 9, no. 1, pp. 1-12, 2018.

[53] M. E. Katt, A. L. Placone, A. D. Wong, Z. S. Xu, and P. C. Searson, "In vitro tumor models: Advantages, disadvantages, variables, and selecting the right platform," Front. Bioeng. Biotechnol., vol. 4, no. FEB, 2016.

[54] S. A. Langhans, "Three-dimensional in vitro cell culture models in drug discovery and drug repositioning," Front. Pharmacol., vol. 9, no. JAN, pp. 1-14, 2018.

[55] C. M. Higuchi, Y. Maeda, T. Horiuchi, and Y. Yamazaki, "A simplified method for three-dimensional (3-D) Ovarian tissue culture yielding oocytes competent to 
599

600

601

602

603

604

605

606

607

608

609

610

611

612

613

614

615

616

617

618

619

[56] T. C. Schulz, "Development and Translation of Stem Cell-Derived Therapies Enabling Technologies for Cell-Based Clinical Translation Enabling Technologies for Cell-Based Clinical Translation," Stem Cells Tranlsational Med., vol. 4, pp. 927931, 2015.

[57] N. Raffel, R. Dittrich, T. Bäuerle, L. Seyler, A. Fattahi, I. Hoffmann, A. Leal-Egaña, M. W. Beckmann, A. R. Boccaccini, and L. Liverani, “Novel approach for the assessment of ovarian follicles infiltration in polymeric electrospun patterned scaffolds," PLoS One, vol. 14, no. 4, pp. 1-12, 2019.

[58] M. Súarez, E. Gómez, A. Murillo, A. Fernández, S. Carrocera, D. Martín, R. Torrecillas, and M. Muñoz, "Development of a novel 3D glass-ceramic scaffold for endometrial cell in vitro culture," Ceram. Int., vol. 44, no. 12, pp. 14920-14924, 2018.

[59] S. V. Murphy and A. Atala, "3D bioprinting of tissues and organs," Nat. Biotechnol., 2014.

[60] Q. Yan, H. Dong, J. Su, J. Han, B. Song, Q. Wei, and Y. Shi, "A Review of 3D Printing Technology for Medical Applications," Engineering, vol. 4, no. 5, pp. 729-742, 2018.

[61] N. Shahrubudin, T. C. Lee, and R. Ramlan, "An overview on 3D printing technology: Technological, materials, and applications," Procedia Manuf., vol. 35, pp. 1286-1296, 2019.

[62] T. D. Ngo, A. Kashani, G. Imbalzano, K. T. Q. Nguyen, and D. Hui, “Additive 
manufacturing (3D printing): A review of materials, methods, applications and challenges," Compos. Part B Eng., vol. 143, no. February, pp. 172-196, 2018.

[63] M. M. Laronda, A. L. Rutz, S. Xiao, K. A. Whelan, F. E. Duncan, E. W. Roth, T. K. Woodruff, and R. N. Shah, "A bioprosthetic ovary created using 3D printed microporous scaffolds restores ovarian function in sterilized mice," Nat. Commun., vol. 8, no. May, pp. 1-10, 2017.

[64] S. S. Suarez and M. Wu, "Microfluidic devices for the study of sperm migration," Mol. Hum. Reprod., vol. 23, no. 4, pp. 227-234, 2017.

[65] B. S. Cho, T. G. Schuster, X. Zhu, D. Chang, G. D. Smith, and S. Takayama, "Passively driven integrated microfluidic system for separation of motile sperm," Anal. Chem., vol. 75, no. 7, pp. 1671-1675, 2003.

[66] Y. Date, S. Takano, H. Shiku, K. Ino, T. Ito-Sasaki, M. Yokoo, H. Abe, and T. Matsue, "Monitoring oxygen consumption of single mouse embryos using an integrated electrochemical microdevice," Biosens. Bioelectron., vol. 30, no. 1, pp. 100-106, 2011.

[67] H. Y. Huang, H. H. Shen, C. H. Tien, C. J. Li, S. K. Fan, C. H. Liu, W. S. Hsu, and D. J. Yao, “Digital microfluidic dynamic culture of mammalian embryos on an Electrowetting on Dielectric (EWOD) chip," PLoS One, vol. 10, no. 5, pp. 1-15, 2015.

[68] H. C. Zeringue, M. B. Wheeler, and D. J. Beebe, "A microfluidic method for removal of the zona pellucida from mammalian embryos," Lab Chip, vol. 5, no. 1, pp. 108-110, 2005. 
[69] S. L. Angione, N. Oulhen, L. M. Brayboy, A. Tripathi, and G. M. Wessel, "Simple Perfusion Apparatus (SPA) for Manipulation, Tracking and Study of Oocytes and Embryos," Fertil Steril, vol. 25, no. 5, pp. 1032-1057, 2016.

[70] F. Yin, Y. Zhu, M. Zhang, H. Yu, W. Chen, and J. Qin, “A 3D human placenta-on-achip model to probe nanoparticle exposure at the placental barrier," Toxicol. Vitr., vol. 54, no. September 2018, pp. 105-113, 2019.

[71] G. Weisgrab, A. Ovsianikov, and P. F. Costa, "Functional 3D Printing for Microfluidic Chips," Adv. Mater. Technol., vol. 1900275, p. 1900275, 2019.

[72] J. E. Sosa-Hernández, A. M. Villalba-Rodríguez, K. D. Romero-Castillo, M. A. Aguilar-Aguila-Isaías, I. E. García-Reyes, A. Hernández-Antonio, I. Ahmed, A. Sharma, R. Parra-Saldívar, and H. M. N. Iqbal, "Organs-on-a-chip module: A review from the development and applications perspective," Micromachines, vol. 9, no. 10, 2018.

[73] M. Haddrick and P. B. Simpson, “Organ-on-a-chip technology: turning its potential for clinical benefit into reality," Drug Discov. Today, vol. 24, no. 5, pp. 1217-1223, 2019.

[74] X. Li and T. Tian, "Recent advances in an organ-on-a-chip: Biomarker analysis and applications," Anal. Methods, vol. 10, no. 26, pp. 3122-3130, 2018.

[75] Y. K. Lee, X. Ran, K. W. H. Lai, V. Y. M. Lau, D. C. W. Siu, and H. F. Tse, "Generation and Characterization of Patient-Specific iPSC Model for Cardiovascular Disease," Methods Mol. Biol., no. 1341, pp. 257-284, 2016.

[76] Y. Li, K. Sallam, P. J. Schwartz, and J. C. Wu, "Patient-Specific iPSC-Based Disease Model 
for Pathogenesis Studies and Clinical Pharmacotherapy," Circ Arrhythm Electrophysiol, vol. 176, no. 5, pp. 139-148, 2017.

666 [77] M. De Almeida, M. Melo, H. H. W. Henning, P. Ferreira, L. Gac, F. Bray, M. B. M. Van Duursen, J. F. Brouwers, T. A. E. Stout, and B. M. Gadella, “Potential Health and Environmental Risks of Three-Dimensional Engineered Polymers," pp. 3-8, 2018.

670

[78] "Micrograph of oviduct of a rat." [Online]. Available: https://instruction.cvhs.okstate.edu/Histology/HistologyReference/HRFemaleRS.

672 htm. 